(Se) former pour enseigner le français à ceux qui ne le parlent pas nativement -

L'École Jules-Ferry, école normale de l'enseignement colonial : une formation pour apprendre à enseigner dans les colonies (1902-1912)

\title{
Gérard Vigner
}

\section{(2) OpenEdition \\ Journals}

\section{Édition électronique}

URL : https://journals.openedition.org/dhfles/4273

DOI : $10.4000 /$ dhfles. 4273

ISSN : 2221-4038

\section{Éditeur}

Société Internationale pour l'Histoire du Français Langue Étrangère ou Seconde

\section{Édition imprimée}

Date de publication : 1 décembre 2015

Pagination : $57-82$

ISSN : 0992-7654

Référence électronique

Gérard Vigner, « L'École Jules-Ferry, école normale de l'enseignement colonial : une formation pour apprendre à enseigner dans les colonies (1902-1912) ", Documents pour l'histoire du français langue étrangère ou seconde [En ligne], 55 | 2015, mis en ligne le 01 janvier 2018, consulté le 26 mars 2023. URL : http://journals.openedition.org/dhfles/4273 ; DOI : https://doi.org/10.4000/dhfles.4273

Ce document a été généré automatiquement le 26 mars 2023.

Tous droits réservés 


\title{
L'École Jules-Ferry, école normale de l'enseignement colonial : une formation pour apprendre à enseigner dans les colonies (1902-1912)
}

\author{
Gérard Vigner
}

1 La Mission laïque française (désormais MLF) est une association créée en juin 1902 à l'initiative d'un certain nombre de personnes appartenant au milieu de l'enseignement, proches des milieux francs-maçons, et soucieuses de faire de l'école un vecteur de diffusion de la langue française et d'un idéal laïque et républicain ${ }^{1}$. Initiative qui, dans cette visée, n'est pas nouvelle. L'Alliance israélite universelle (AIU), fondée à Paris en 1860 , se consacrait déjà à l'éducation et avait ouvert de nombreuses écoles dans le bassin méditerranéen ${ }^{2}$, la première ouverte à Tétouan en 1862, l'Alliance française (AF) pour sa part, créée en 1883, avec Pierre Foncin comme premier président, alors secrétaire général de l'AF, s'était donnée aussi pour projet de diffuser la langue et la culture françaises et de bâtir un réseau d'établissements et d'instituts en différents endroits du monde ${ }^{3}$. Mais l'AIU était d'abord soucieuse d'apporter son appui à des populations juives laissées en déshérence, de contribuer à leur émancipation en diffusant la culture et la langue françaises, alors que pour l'AF, l'école n'était qu'un vecteur, parmi d'autres, de diffusion et d'influence et ne manquait pas parfois de subventionner des écoles congréganistes, ce qui ne pouvait que choquer les milieux laïques. La MLF, au contraire, souhaite diffuser un idéal laïque et républicain par le moyen de l'école, dans les colonies et à l'étranger et la langue française en sera le vecteur de diffusion.

2 À cet effet, la MLF décide de créer un dispositif de formation, qui va être nommé significativement, l'École Jules-Ferry (désormais EJF), hommage évident au fondateur de l'école laïque, chargé de former des enseignants, des missionnaires laïques devant 
exercer dans les écoles publiques des colonies ou dans des écoles de la Mission à l'étranger. L'EJF a fait l'objet d'un nombre limité d'études, dans la mesure certainement où cette instance de formation ne fut ouverte que d'octobre 1902 à juin 1913, et ne constitua qu'un moment de l'histoire de la MLF${ }^{4}$. Au-delà du cadre constitué par l'EJF, mieux connu aujourd'hui, nous voudrions nous intéresser au programme de formation et aux conditions de sa mise en place à destination des futurs " instituteurs coloniaux " pour reprendre l'expression utilisée par les fondateurs de l'école. Question d'autant plus intéressante que cette formation fut la seule spécialement dédiée à cette tâche. Si en effet les missionnaires religieux étaient préparés en métropole à leur mission d'évangélisation, l'école en constituant un des volets d'intervention, rien de tel en revanche pour les enseignants appelés à exercer dans les réseaux d'écoles indigènes qui se mettaient en place à ce moment-là dans les territoires des colonies. L'instruction ne rentrait pas dans les attributions du ministère des Colonies, récemment créé, en 1894, qui en avait laissé la responsabilité aux gouverneurs.

\section{La création de la Mission laïque française}

La phase proprement militaire de la conquête coloniale s'achève dans la fin des années 1880. L'armée se désengage progressivement de la gestion des territoires, y compris parfois de ses missions d'enseignement comme dans le Haut-Sénégal par exemple ${ }^{5}$, et souhaite passer le relais à un personnel civil. Les autorités françaises se rendent compte ainsi qu'il convient de disposer d'un personnel spécialement formé à cet effet. La professionnalisation des carrières coloniales apparaît comme une nécessité. L'École libre des Sciences politiques ouvre des cours coloniaux dès 1886, avec comme objectif la formation de futurs administrateurs, mission dont elle va d'ailleurs être progressivement privée par la création de l'École coloniale en 1889 qui va être chargée de former les administrateurs coloniaux, désormais constitués en fonctionnaires civils de l'État (P. Singaravélou 2011). Un enseignement commercial colonial se met en place dans les écoles supérieures coloniales dès 1890 . Une École pratique coloniale est encore créée au Havre en 1892. Un très large mouvement se dessine de la sorte destiné à fournir aux colonies le personnel d'encadrement et les spécialistes dont elles vont avoir besoin. Seul le domaine de l'instruction ne bénéficie pas d'un engagement particulier alors qu'un peu partout dans les colonies, dans la dernière décennie du XIX ${ }^{e}$ siècle, se pose la question de la création d'une école indigène, de la nécessité ou non d'y enseigner le français, les congrès coloniaux, nombreux en ce temps-là, ouvrant de nombreux débats sur cette question ${ }^{6}$. Quel type d'école mettre en place? Quel plan de formation adopter? Quels contenus d'enseignement envisager? Jusqu'où aller dans une formation dont certains milieux coloniaux redoutent les effets à terme? Telles sont les questions pour lesquelles on ne dispose pas encore de réponses, faute d'expérience en la matière. Tout est à construire.

4 Présentes dans les territoires colonisés souvent bien avant l'arrivée du colonisateur, les missions religieuses avaient déjà ouvert des écoles, mais écoles dans lesquelles les visées d'instruction et d'évangélisation étaient étroitement mêlées. En certains endroits, les autorités coloniales, peu compétentes en ce domaine et peu intéressées par la question, il faut bien le reconnaître, avaient confié aux missions le soin de mettre en place les premières bases d'une école indigène, ainsi de la Côte d'Ivoire et de la Société des Missions africaines de Lyon en 1896. Les milieux laïques et les milieux 
francs-maçons, présents aussi dans les colonies, ne pouvaient que déplorer une telle mainmise des réseaux missionnaires, très nombreux et très puissants, sur l'action éducative dans les colonies ${ }^{7}$, alors même que dans la suite de l'affaire Dreyfus, l'anticléricalisme, après des tentatives réussies d'apaisement engagées en métropole, connaissait un regain de vigueur. Inquiets, les milieux laïques, témoins parfois d'une mauvaise volonté des missionnaires à apprendre le français à leurs élèves, alors que l'on enseignait le latin dans certaines écoles d'Indochine par exemple, se devaient de réagir et créèrent donc le symétrique des missions religieuses, sous l'impulsion notamment de Pierre Deschamps (1873-1958) ${ }^{8}$, directeur de l'enseignement à Madagascar qui sut trouver chez Pierre Foncin, ancien président de l'Alliance française, devenu depuis son secrétaire général, une oreille attentive.

5 Une assemblée générale constitutive se tient le 2 juin 1902. Et Pierre Foncin est élu comme premier président, fonction qu'il occupera jusqu'en 1903. Est ainsi constituée : «La Mission laïque, association pour la propagation de l'enseignement laïque aux colonies et à l'étranger ». Dans les statuts adoptés le 8 juin 1902, il est précisé :

Article 7

Pour la formation des instituteurs et des institutrices qui se destinent à l'enseignement, soit dans les écoles publiques des colonies, soit dans les établissements créés ou encouragés par la Mission laïque, il est fondé à Paris une École normale, relevant directement de l'Association et appelée École Jules-Ferry.

Sur la deuxième de couverture de la revue de l'Association est rappelé encore :

La Mission laïque prépare à l'École Jules-Ferry, fondée et entretenue par elle, des instituteurs coloniaux et des missionnaires laïques. Elle soutient, moralement et matériellement, des écoles laïques françaises aux colonies et à l'étranger. Elle crée et dirige des écoles laïques relevant directement d'elle dans les pays d'influence française.

7 Ouverte en octobre 1902, l'École Jules Ferry comprend dans son comité de direction, dont la composition pourra varier, Laurent Devina, directeur de l'école normale d'instituteurs d'Auteuil, Clotaire Baudrillard et Henri Ferrier, tous deux inspecteurs primaires du département de la Seine, tous trois professionnels reconnus de l'enseignement primaire, Henri Ferrier ayant lui-même exercé en Algérie, à la Martinique et à Pondichéry. Marie-Jeanne Potel, professeur à l'école Sophie-Germain de Paris jouera aussi un rôle important dans l'organisation et le suivi de la formation. Le Conseil d'administration de la MLF fait d'ailleurs apparaître une très forte présence d'enseignants exerçant dans le département de la Seine.

8 Pour mieux faire connaître l'action de la MLF auprès du public et en même temps pour servir de bulletin de liaison entre les personnels est créée en 1904 une revue, la Revue de l'enseignement colonial, organe des écoles laïques françaises hors de France. Le premier numéro est publié en janvier-février 1904. On y annonce notamment, pour la partie consacrée à l'EJF, le programme de l'année à venir, les nominations dans les postes pour les boursiers de l'année précédente, on annonce les mariages mais aussi parfois les décès, enfin certains boursiers racontent le voyage qui les a conduit de Marseille à leur nouveau poste.

L'école ainsi fondée dispose quelque part de deux références, d'une part l'«École normale des Instituteurs orientaux » (ENIO), fondée par l'Alliance israélite universelle, en 1867, chargée de former les enseignants appelés à exercer dans les écoles progressivement ouvertes dans le pourtour du bassin méditerranéen. Les responsables de l'AIU s'étaient en effet rendu compte de la nécessité de disposer d'un personnel 
spécialement formé à cet égard, compte tenu des particularités du contexte. L'Alliance française avait à un moment réfléchi à la possibilité d'ouvrir elle aussi une école de formation pour enseignants devant partir exercer à l'étranger et notamment dans les colonies. Mais le projet n'avait pas abouti. Elle avait en revanche ouvert les premiers cours d'été, à l'initiative de Ferdinand Brunot en $1894^{\circ}$. Enfin on ne pouvait ignorer l'existence du réseau déjà pleinement opérationnel en France des écoles normales d'instituteurs et d'institutrices chargé de la formation initiale des enseignants ayant à exercer dans l'école primaire. Le directeur de l'EJF dans les premières années, Laurent Devina, est en même temps directeur de l'école normale d'instituteurs d'Auteuil.

Mais l'EJF a un statut particulier. Elle est un dispositif de formation non officiel, relevant d'une initiative privée, même si tous les concepteurs sont membres du ministère de l'Instruction publique. Elle dispense un complément de formation à destination de personnels issus des écoles normales d'instituteurs.

\section{Le recrutement du personnel}

11 Peuvent postuler pour cette formation, soit des instituteurs titulaires du Brevet supérieur et du CAP (Certificat d'aptitude pédagogique), les hommes, de plus, doivent avoir satisfait aux obligations militaires, soit des instituteurs en exercice à Madagascar et en congé en métropole. Les épouses d'instituteur sont admises en position d'auditeur libre, il était fréquent en effet, dans les colonies, de recourir aux services bénévoles des épouses d'instituteur pour toutes sortes d'activités périscolaires (il en allait d'ailleurs de même pour les épouses de pasteur dans les écoles de mission protestantes). On acceptait aussi des ménages, c'est-à-dire des couples d'instituteurs, l'école primaire française en ce temps-là favorisant le mariage entre enseignants normaliens, ce qui permettait ainsi à un couple de postuler pour des postes doubles, nombreux dans les villages en ce temps-là et où l'on distinguait soigneusement écoles de filles et écoles de garçons. Ainsi de M. et de Mme Hyvernaud, enseignants au Poinçonnet dans l'Indre, inscrits dans la promotion 1903-1904. Mais dans tous les cas, les enseignants recrutés étaient passés par les écoles normales d'instituteurs et d'institutrices. L'examen des dossiers de recrutement l'atteste. La titularisation préalable en France était en même temps la condition de leur rattachement au cadre métropolitain au moment de leur départ. Les colonies n'avaient pas en effet compétence pour recruter des personnels susceptibles d'être titularisés dans les cadres métropolitains.

Les candidats devaient adresser une lettre de demande d'admission auprès du président de la MLF. L'avis de l'inspecteur primaire et de l'inspecteur d'académie était sollicité. Au-delà des critères d'appréciation d'ordre moral et professionnel, il s'agissait de savoir s'il était possible de remplacer l'enseignant dans son poste. L'instituteur devait signer un engagement à servir pendant au moins trois ans dans les écoles publiques des colonies ou dans celles de la MLF. Il était placé en position de congé administratif, en externat gratuit, et touchait une bourse mensuelle de 125 fr. (1905-1906). Pour autant rien n'était acquis pour ce qui relevait de son affectation. Il devait présenter, en fin d'année de formation, sa candidature auprès du ministère des Colonies, avec l'appui de la MLF, les retards de nomination l'obligeant parfois à reprendre son poste dans son affectation première. 


\section{La formation}

L'objectif n'est pas de former des instituteurs au sens général du terme, ils le sont déjà, dans leurs écoles normales d'origine, mais des instituteurs coloniaux et des missionnaires laïques :

L'enseignement qui y est donné est conçu de manière que les futurs instituteurs coloniaux puissent être à la fois des éducateurs au courant des conditions de pédagogie indigène, et des auxiliaires éclairés de l'œuvre colonisatrice; aussi son programme comprend, en outre de l'étude des méthodes de l'enseignement colonial, des cours d'agriculture coloniale, de médecine pratique, de langues indigènes, de travaux techniques; il est essentiellement tourné vers les applications pratiques, en vue de l'action immédiate. ( $R E C, \mathrm{n}^{\circ} 1$, janvier 1904, p. 30)

Les élèves sont répartis en sections pour répondre aux demandes des colonies. On n'oubliera pas en effet que certains territoires adressent des subventions à la MLF pour répondre à leurs besoins de formation en matière de personnel qualifié. La première section ouverte le fut à la demande de Galliéni, gouverneur général de Madagascar de 1896 à 1905, puis viendront l'Afrique noire, l'Asie-Océanie plus particulièrement orientée vers l'Indochine, enfin en 1912, pour répondre à la demande du maréchal Lyautey, nommé résident général au Maroc, on projette d'ouvrir une section Maroc ${ }^{10}$. Mais l'approche de la guerre et l'impossibilité dans laquelle se trouve la MLF de recruter une nouvelle promotion pour 1913-1914, ne permettra pas de donner consistance à ce projet.

La formation comprend :

- des cours généraux: pédagogie, agriculture coloniale, médecine et hygiène, enseignement technique

- des cours spéciaux: section africaine (arabe littéraire, institutions musulmanes, sociologie appliquée à la colonisation, races et populations du Soudan); section malgache (malgache, conférences, pratiques, administration) ${ }^{11}$

- des travaux pratiques : travail manuel sur le bois et le fer pour les hommes, dessin appliqué et travaux pratiques pour les femmes, broderie, coupe, couture ${ }^{12}$

- des stages en hôpital auprès des personnels infirmiers pour les institutrices

- des conférences : École des Hautes études sociales, conférences données par des officiers, des administrateurs coloniaux, des enseignants en congé, etc.

- des cours de langue : (malgache, arabe vulgaire, wolof, annamite, selon les années et les sections). 


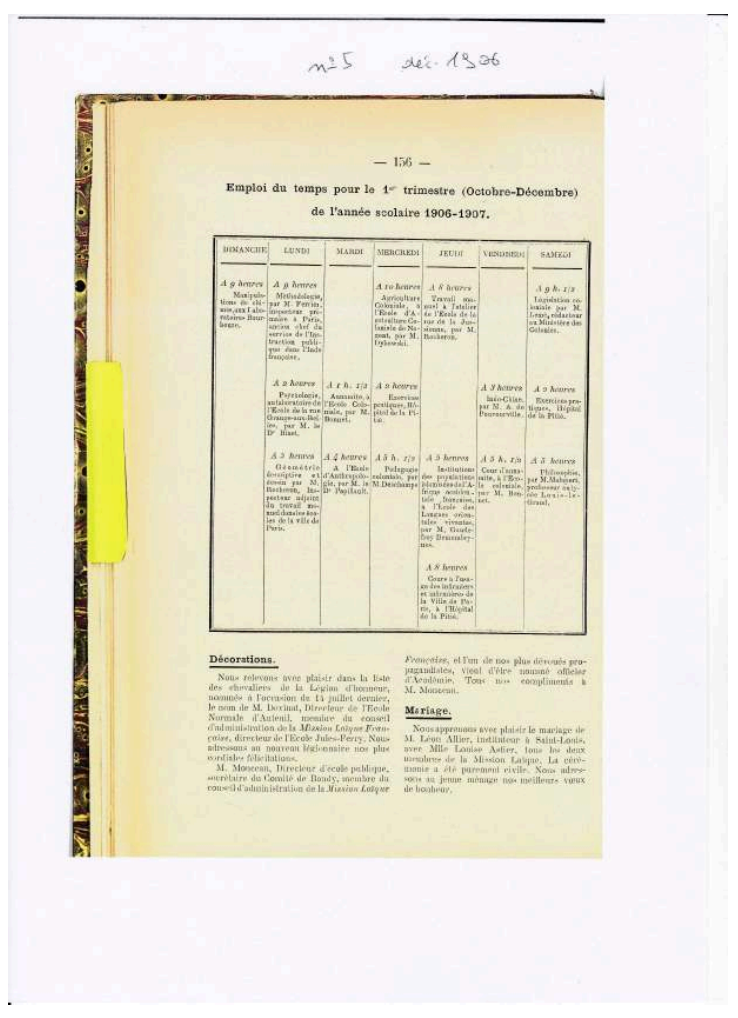

Revue de l'Enseignement colonial, n 5, 1906. formation dans les locaux même de la Mission, ainsi qu'en différents lieux, dans Paris et d'élaboration des savoirs coloniaux ou du moins qui peuvent les éclairer. Ainsi de cet emploi du temps (voir supra) qui fait se rendre les élèves le dimanche aux Laboratoires Bourbouze, société à vocation philanthropique, installée rue de Jouy, dispensant des cours en physique et en chimie, pour des manipulations de chimie, puis le lundi au laboratoire de psychologie de l'école de la rue de la Granges-aux-Belles, pour y rencontrer le docteur Binet, le célèbre psychologue, à l'École coloniale, à l'École d'anthropologie, à l'École d'agriculture coloniale à Nogent-sur-Marne, à l'atelier de l'école de la rue de la Jussienne pour le travail manuel, à l'École des Langues' o, pour des cours d'annamite et à l'hôpital de la Pitié-Salpétrière pour des stages d'infirmières. Des sources d'information, et de formations pratiques, variées recueillies auprès des spécialistes du domaine et dans leur lieu d'exercice. Un dispositif de formation léger, qui prend appui sur les institutions existantes et qui évite pour l'EJF l'inconvénient de se constituer en établissement spécifique, doté de locaux propres et d'un personnel dédié, ce que les ressources disponibles ne permettaient pas. Programme lourd, qui se traduit par un emploi du temps particulièrement chargé, puisque l'on travaille du lundi au dimanche, sur 10 mois environ.

$$
\begin{aligned}
& \text { for } \\
& \text { d'é } \\
& \text { em } \\
& \text { Bo } \\
& \text { coub } \\
& \text { lab } \\
& \text { d'éc } \\
& \text { des } \\
& \text { De } \\
& \text { spéc } \\
& \text { qui } \\
& \text { se } \\
& \text { dédié } \\
& \text { trad } \\
& \text { au }
\end{aligned}
$$




\section{Des principes}

Même si cette institution porte le nom d'«école normale", en référence aux institutions chargées de former les futurs instituteurs, l'ENJF, par son statut, comme par ses contenus de formation ne saurait être confondue avec les établissements chargés de préparer leurs élèves aux fonctions d'instituteur ou d'institutrice. Établissements publics chargés de dispenser un complément d'instruction et de mettre en place une formation professionnelle, les écoles normales consacraient en réalité l'essentiel de leurs efforts à des compléments d'instruction qui sont proches de ceux dispensés dans les lycées. L'âge d'admission est d'ailleurs abaissé à 15 ans en 1880 . L'examen de la répartition des matières d'enseignement le fait clairement apparaître :

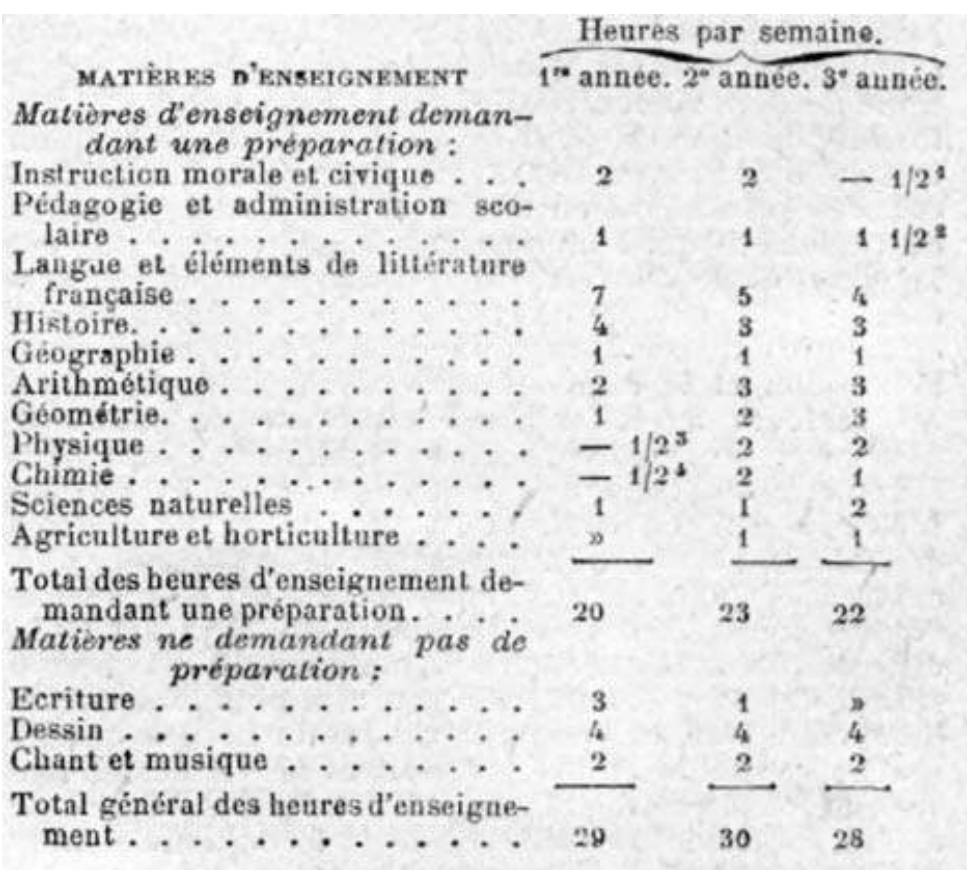

1. Une beure par semaine pendant un semestre. - 2. Deux heures pendant un semestre, une heure pendant l'autre. -3 . Une heure pendant un somestre. - 4. Une heure pendant l'autre semestre. 


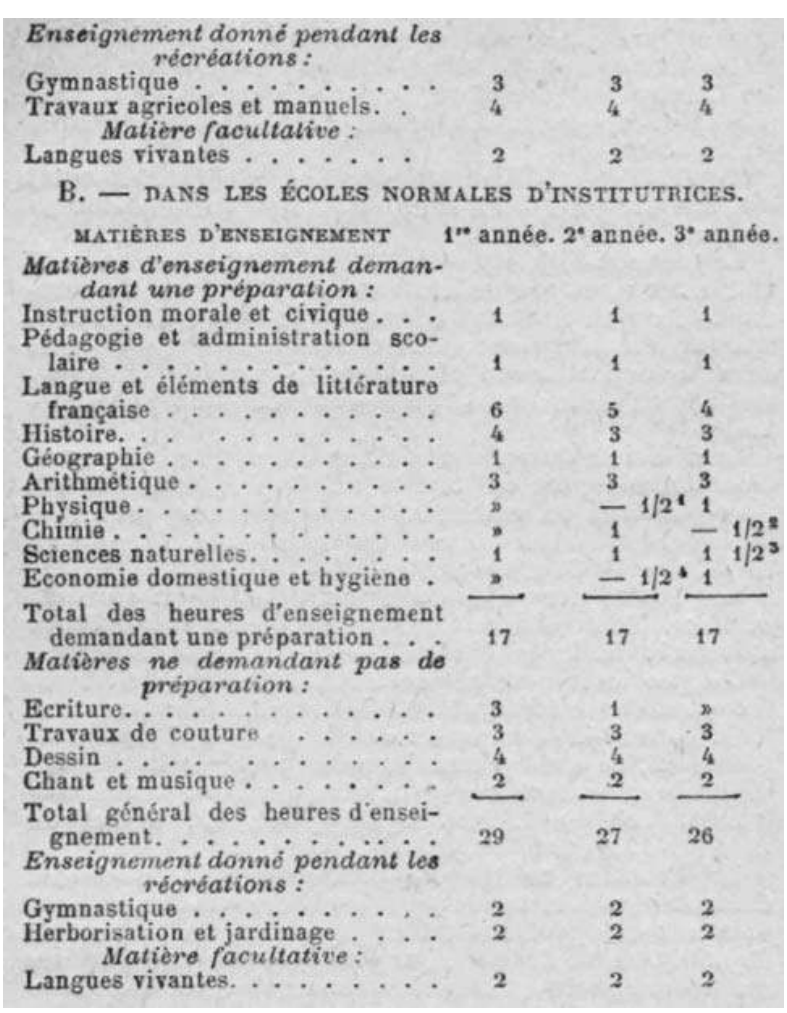

Extrait du Dictionnaire pédagogique, 1911, article Normales primaires (écoles).

Les matières d'enseignement sont celles, à quelques particularités près, que l'on peut retrouver dans les lycées de l'époque. L'enseignement y est dispensé par un corps d'enseignants spécialisés, les professeurs d'école normale, formés à l'École normale supérieure de Saint-Cloud, créée en 1882, établissement d'enseignement supérieur qui constituera en quelque sorte l'université de l'enseignement primaire et dote cet ordre d'enseignement d'un cycle complet de formation, distinct de l'enseignement secondaire qui prenait appui sur l'Université. Rien de tel pour les boursiers de l'EJF qui reçoivent un complément de formation professionnelle pour exercer dans d'autres territoires et auprès de populations scolaires radicalement nouvelles.

Même si l'enseignement du français par la méthode directe est régulièrement évoqué, notamment par Henri Ferrier qui fait part de son expérience en la matière, mais aussi, selon les années par d'autres intervenants, tel $M$. Delale chef du service de l'enseignement dans l'Inde française, pour 1907-1908, cette formation est souvent, pour certaines années, incluse dans un ensemble intitulé "pédagogie coloniale» qui comprend : « des notions sur l'organisation scolaire aux colonies, l'enseignement des indigènes, les programmes et les méthodes, la méthode directe pour l'enseignement de la langue française » $(R E C, 5$, octobre 1907, p. 157). Mais en 1906-1907, on distingue la « méthodologie », proposée par Henri Ferrier, de la " pédagogie coloniale » proprement dite, qui sera présentée par Pierre Deschamps :

M. Deschamps, chef du service de l'enseignement à Madagascar, en congé, se propose d'examiner, avec les élèves de l'École Jules-Ferry, comment peut être résolu ce problème si intéressant et si complexe de l'Éducation d'une race par une autre, et en particulier, de l'éducation méthodique des indigènes des Colonies par des instituteurs d'origine européenn $\mathrm{e}^{13}$. (REC, $\mathrm{n}^{\circ} 1$, janvier 1906, p. 32)

21 L'enseignement de la langue française ne constitue pas le cœur de cette formation, bien loin de là, alors même qu'il s'agit de diffuser une langue par des méthodes différentes 
de celles qui prévalaient dans les écoles de France, d'où provenaient les boursiers de l'EJF. Sur un échantillon examiné de 32 dossiers de recrutement, seuls deux boursiers ont une expérience d'enseignement à l'étranger, l'un, Henri Caillarec, ayant enseigné le français à l'école Berlitz aux Pays-Bas, une autre, Agnès Jarzuel ayant enseigné en Angleterre, en Allemagne et à Madagascar. À l'évidence le fait d'avoir une expérience de l'enseignement du français auprès de publics étrangers ne constituait pas un critère déterminant de sélection des candidats. Henri Ferrier, se fondant sur son expérience de l'enseignement dans les colonies, signale tout l'intérêt que présente la méthode directe. Dans le $\mathrm{n}^{\circ} 4$ de la REC (juillet-août 1904, p. 104), il publie un article intitulé " L'Enseignement du français aux colonies par la méthode directe» qui est plus le témoignage d'une expérience vécue que d'une analyse plus fondamentale des procédés à mettre en œuvre. Il conclut d'ailleurs plein d'optimisme :

On peut dire que grâce à la méthode directe, le français est devenu, pour les enfants de douze à treize ans, ayant régulièrement fréquenté l'école, une deuxième langue maternelle.

Mais Henri Ferrier ne fait que reprendre ici la doxa qui est en train de se constituer pour définir un enseignement du français dans les colonies, en opposition à la méthode de traduction, lancée par Jean Dard au Sénégal en 1817, ou à celle d'un enseignement exclusivement dispensé en langues indigènes, souvent adopté par les missions religieuses.

Plus intéressant, et expression plus nuancée de la vision de ce pourrait être un enseignement $d u$ français, est le compte rendu établi par un des rapporteurs à l'occasion du Congrès de l'enseignement colonial et de l'enseignement laïque à l'étranger, qui se tient à Marseille dans l'été 1906, à l'initiative de la MLF. Parmi multiples commissions qui s'y tiennent, une est consacrée aux «Buts et méthodes de l'enseignement du français aux indigènes des colonies » :

Le rapporteur de cette importante question n'a pas encore adressé au secrétariat général du Congrès les conclusions auxquelles il est arrivé. Mais d'ores et déjà on peut dire qu'il sera proposé au Congrès d'émettre les vœux suivants qui n'ont pas reçu leur forme définitive $: 1^{\circ}$ que le but de l'enseignement du français ne soit pas la substitution de la langue française à la langue maternelle des indigènes de nos colonies $; 2^{\circ}$ que pour des raisons d'ordre politique et d'intérêt pratique, le français soit enseigné dans toutes les écoles des colonies, même dans les écoles du premier degré $; 3^{\circ}$ que la dénomination d'école ne soit pas donnée par les arrêtés et règlements scolaires aux établissements dans lesquels le français n'est pas enseigné ; $4^{\circ}$ que le français enseigné dans les écoles soit un français usuel, « réduit », mais correct; $5^{\circ}$ que la méthode employée soit la méthode directe, d'abord exclusivement, puis associée à l'enseignement de la lecture, et enfin à la méthode dite de «traduction "; $6^{\circ}$ que dans les écoles du $2^{\text {ème }}$ degré et à plus forte raison dans les $3^{\text {èmes }}$ degré l'enseignement soit, en partie au moins, donné en français ; que dans les écoles, la langue française, après avoir été un but devienne un moyen. (REC, $\mathrm{n}^{\circ} 4$, août 1906, p. 100)

Citation un peu longue mais qui mérite d'être signalée pour pointer des choix qui ne donnent pas à la langue française une position de monopole dans la scolarisation des enfants indigènes. Le malgache était déjà enseigné à Madagascar à côté du français, le vietnamien dans un certain nombre d'écoles d'Indochine et l'arabe en Tunisie encore. On signalera la préférence pour l'enseignement d'un «français réduit », vieux projet lancé par l'Alliance française, mais qui ne donna pas lieu à une réalisation effective ; 
enfin la méthode dite de "traduction », référence à l'autre méthode d'enseignement, en opposition à la méthode directe, est ici évoquée.

Il n'est peut-être pas inutile encore de s'attarder un bref moment sur les encarts financés par les éditeurs de manuels scolaires et présents dans la REC. Deux méthodes y sont largement et régulièrement présentées par les éditions Armand Colin, la "Méthode Carré » et la "Méthode Machuel », deux méthodes spécialement destinées aux élèves indigènes des colonies et déclinées en différents matériels :

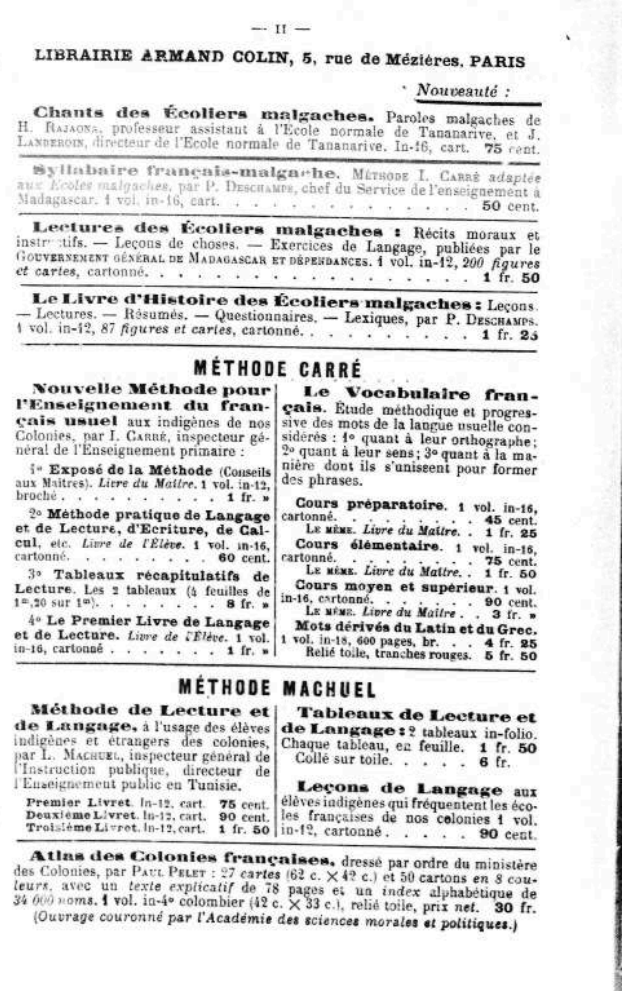

La "Méthode Carré » est héritière des principes de la "méthode maternelle » dont Irénée Carré (1829-1909) présente les principes dès 1888, à destination d'abord des enfants de Basse-Bretagne, puis des élèves des colonies. Louis Machuel (1848-1922) s'était en revanche illustré par la volonté de promouvoir en Tunisie un enseignement qui prenne appui sur la langue des élèves, qu'il s'agisse de l'arabe ou de l'italien. Dans les deux cas cependant, et même si les deux auteurs sont de sensibilité tout à fait différente au plan pédagogique et certainement intellectuel, ces deux méthodes relèvent de la méthodologie directe ${ }^{14}$, ce qui est en cohérence avec les principes qui prévalent, dans la plus grande partie des colonies, en matière d'enseignement du français. On notera cependant, en haut de la page, la mention d'un syllabaire francomalgache, issu de la méthode Carré et adapté aux écoles malgaches par Pierre Deschamps, qui exerça à Madagascar comme directeur de l'Enseignement de 1898 à 1906, qui témoigne d'une autre politique plus favorable, dans certains cas, à l'enseignement des langues indigènes.

Pour autant, un examen rapide de l'emploi du temps montre combien ces questions pédagogiques et notamment celles se rapportant à l'enseignement du français, points de débat pourtant majeurs dans les différents territoires des colonies, occupent une place restreinte. La dimension pratique de la formation y occupe une place importante, pour tout ce qui concerne le travail manuel, ainsi que la présentation des colonies, celle 
de la législation en cours, à côté des cours d'agronomie tropicale. Tous ces éléments dessinent le portrait d'un instituteur ou d'une institutrice qui est plus un missionnaire, une sorte de pionnier, capable d'exercer sa mission dans des écoles de brousse les plus reculées, disposant d'un savoir-technique en matière de travail manuel et de nombreuses ressources d'intervention en matière de soin, grâce aux stages en milieu hospitalier. Ainsi du programme de " Médecine et d'hygiène » détaillé en :

1) Éléments de médecine générale.

2) Petite chirurgie. Éléments de chirurgie générale. Les maladies vénériennes.

Affections spéciales.

3) Hygiène générale.

4) Hygiène coloniale.

La lecture qui est ainsi donnée de l'espace colonial est celle d'un monde dont l'altérité, parfois inquiétante avec son long cortège de maladies, doit être apprivoisée. L'école prend place dans un espace politique à la consolidation duquel elle doit participer, avec une réponse qui est celle de pédagogues républicains éclairés, laïques, à l'écoute des besoins des populations :

Cet enseignement, ce qui le caractérise d'abord, c'est sa souplesse. Il ne s'agit pas d'imposer aux gens une méthode, un programme qu'on croit parfaits pour les avoir vus réussir ailleurs ; mais bien plutôt de leur donner ce qu'ils souhaitent, de se plier à leurs besoins, de se conformer à leurs dispositions, dans la mesure du possible, d'agir dans leur intérêt. (M. Fort, Bulletin de la Mission laïque française, 4, avril 1914)

Cette thématique de la nécessaire adaptation de l'enseignement au contexte de son implantation et de son accomplissement sera constamment reprise, au moins jusqu'à la Deuxième Guerre mondiale et constituera le thème du Congrès international de l'enseignement dans les colonies et les pays d'outre-mer, qui se tient parallèlement à l'Exposition coloniale de Paris en 1931.

On forme bien des missionnaires qui, à l'image des religieux, iront ouvrir en brousse des stations, c'est-à-dire des lieux support non point de l'évangélisation, mais d'une mission civilisatrice et pourront de la sorte intervenir dans tous les domaines de conquête des esprits. De fait, les postes dans lesquels ces boursiers seront affectés ne correspondront pas forcément à cette conception de l'instituteur colonial pionnier, mais plus à celle d'un professionnel éclairé qui pourra exercer dans des fonctions de responsabilité. Ainsi, Sosthène Penot, formé dans la promotion Paul Bert (1904-1905), dans la section de Madagascar, est affecté à l'école normale Le Myre-de-Vilers de Tananarive ${ }^{15}$, affectation qui semble plus privilégier la compétence du spécialiste de l'enseignement dans les colonies que celle de l'instituteur de brousse.

\section{Un désengagement rapide}

31 Alors même que l'EJF est très tôt mise en place, portée par le volontarisme de ses créateurs, alors que la Mission laïque fait de l'enseignement dans les colonies un axe majeur de son action, cet élan semble connaître dès 1906 un net ralentissement, et ceci pour plusieurs raisons.

Dès 1906 en effet, un certain nombre de responsables de la Mission considèrent que l'Orient, au sens très large du terme, doit constituer un champ d'intervention dans lequel la Mission a un rôle à jouer. Le nouveau président, Alphonse Aulard, professeur à la Sorbonne, qui préside aux destinées de la Mission laïque de 1906 à 1912, peu intéressé par le monde des colonies, à la différence de ses prédécesseurs, Pierre Foncin, 
Eugène Etienne ou Gaston Doumergue, s'intéresse plus particulièrement au développement de l'enseignement laïque en Orient. Des lycées vont être ouverts, à Salonique en 1906, au Caire en 1908, à Alexandrie en 1909, à Beyrouth en 1910, établissements qui vont progressivement absorber l'intérêt des responsables de la Mission. En même temps, cette nouvelle orientation privilégie l'enseignement secondaire, domaine de formation plus proche la culture professionnelle des responsables de la Mission. Significativement, la revue de la Mission modifie son titre qui devient en juin 1907 le Bulletin de la Mission laïque française - Revue de l'enseignement colonial. L'identité institutionnelle l'emporte sur l'engagement initial dans les colonies.

Au-delà de ces changements d'orientation, l'impossible pérennisation des ressources pour financer le fonctionnement de l'EN constitue un facteur de fragilité supplémentaire. Il faut s'en remettre à chaque fois au bon vouloir des gouverneurs des colonies, aux subventions du ministère des Colonies pour financer les bourses attribuées aux enseignants en formation ${ }^{16}$. La Mission laïque, si elle bénéficie d'une reconnaissance de fait de la part des autorités de l'État, elle fut reconnue d'utilité publique le 21 août 1907, reste pour autant une association privée qui dépend de la bonne volonté de ses adhérents et des ressources qui y sont associées ${ }^{17}$. Le nombre d'enseignants susceptibles d'être formés chaque année reste limité, fort éloigné des besoins des colonies en personnel spécialisé.

Les gouverneurs des colonies, disposant de faibles ressources financières, et devant sur leur budget propre financer la totalité de leurs dépenses civiles et donc celles liées à l'action éducative, ne sont en fait que médiocrement intéressés par un personnel venu de métropole et auquel il faudra verser toutes sortes d'indemnités complémentaires, prendre en charge les frais de voyages lors des congés statutaires. Si paradoxal que cela puisse paraître, les personnels exerçant dans les écoles de missions religieuses, ont souvent la préférence des autorités coloniales dans les territoires pour la faiblesse de leurs coûts par rapport au budget de la colonie. De fait le ministère des Colonies ne voulut jamais s'engager officiellement auprès de l'EJF pour lui confier la tâche de former les instituteurs dont les colonies avaient besoin. Certaines années l'EJF est parfois obligée de renouveler l'appel à candidature pour la participation au stage de l'école, on signale ainsi des bourses non attribuées en 1909. À l'évidence, le départ pour les colonies, au moins par le biais de l'EJF, n'a pas suscité l'élan attendu.

En effet, tous les enseignants métropolitains partant pour les colonies ne passent pas loin de là, par la filière de l'ENJF. Le cas de la mère de Marguerite Duras, Marie Donnadieu, l'héroïne, depuis célèbre, du roman Un Barrage contre le Pacifique, est significatif à cet égard. Née à Fruges, dans le Pas-de-Calais en 1877, elle entre à l'école normale d'institutrices de Douai, puis devient institutrice dans l'école d'un petit village, Rexpoede, avant d'être mutée dans une école de Dunkerque. Elle demandera à partir pour l'Indochine et sera nommée en mars 1905 institutrice à l'école municipale de jeunes filles de Saïgon. Elle connaîtra en Indochine une vie familiale et une carrière compliquées, Hanoï, Pnom-Penh, Ving Lon, Sadec, ponctuée de différents allers et retours vers la France, dans tous les cas loin, bien loin de l'École Jules Ferry qu'elle n'a jamais connue ${ }^{18}$. Et son cas est tout sauf isolé, même si le nombre d'enseignants métropolitains envoyés dans les colonies reste limité, si on le compare à celui des enseignants indigènes que les autorités coloniales vont s'efforcer de former le plus rapidement possible ${ }^{19}$. 
En fait les autorités coloniales, sur la sollicitation d'ailleurs du ministère des Colonies, vont progressivement ouvrir dans les territoires des colonies des écoles normales destinées à former un personnel indigène, à la fois considéré comme plus proche des populations et surtout rémunéré de façon infiniment plus modeste ${ }^{20}$. Signalons l'école normale de Saïgon ouverte en 1874, le collège Alaoui de Tunis en 1884, l'école normale Le Myre-de-Vilers, à Tananarive en 1897, l'école normale de Saint-Louis en 1903, qui deviendra par la suite l'école normale William Ponty, l'école des moniteurs de Montravel en Nouvelle-Calédonie en 1913. Si limités qu'aient pu être au début les effectifs d'instituteurs indigènes ainsi formés, dans des conditions d'ailleurs peu satisfaisantes pour ce qui est de la qualité de la formation, pour autant la concurrence était forte et l'école normale Jules Ferry, avec ses promotions modestes, ne pouvait prétendre couvrir à elle seule les besoins en personnels spécialement formés pour enseigner dans les colonies.

Enfin, la loi militaire qui en 1913 porte à trois ans la durée du service militaire, conduit à conserver une année de plus dans les casernes les appelés de 1911 et tarit en partie le recrutement. Les inspecteurs d'académie refusent enfin de donner l'exeat aux instituteurs qui demandaient leur inscription à l'école. La promotion de 1912-1913 sera la dernière de l'EJF.

\section{Pour conclure}

La politique de formation des instituteurs coloniaux engagée par la MLF ne fut pas reconduite au lendemain de la guerre de 1914-18. Des raisons circonstancielles, nous l'avons vu plus haut, peuvent expliquer ce choix. Mais on doit tenir compte aussi de raisons plus fondamentales. La France sort épuisée du premier conflit mondial et n'a plus l'énergie nécessaire pour poursuivre une politique de conquête et d'influence ${ }^{21}$. Une vision gestionnaire des territoires de l'empire se met en place, et à des missionnaires, venus de métropole, portés par un idéal laïque et civilisationnel, on préfère des professionnels, rémunérés au moindre coût, les maîtres indigènes. À peu près partout installée, l'école indigène, même si elle ne scolarise qu'un nombre très faible d'élèves, dispose de ses propres programmes, de ses manuels, de son personnel d'enseignement et d'encadrement.

Lorsque la guerre de 1914 éclate, le ministre de l'Intérieur de l'époque Louis Malvy publie un décret, le 2 août 1914, qui suspend l'exécution des décrets de fermeture des congrégations pris en application des lois de 1901 et de 1904. L'Union sacrée appelle à la réconciliation avec le monde catholique et la paix revenue, on ne reviendra pas sur ces mesures d'apaisement. Le combat pour la laïcité, présent encore dans les esprits, ne revêt plus le même sens et cette disjonction entre missionnaires religieux et missionnaires laïques dans le domaine de l'éducation perd de son intensité. 


\section{BIBLIOGRAPHIE}

Revue de l'enseignement colonial, organe des écoles laïques françaises hors de France, 1904-1907, devenue Bulletin de la Mission laïque française - Revue de l'enseignement colonial, 1907-1914.

ADLER, Laure (1998). Marguerite Duras. Paris : Gallimard, rééd. Folio.

BEZANÇON, Pascale (2002). Une colonisation éducatrice ? L'expérience indochinoise (1860-1945). Paris : L'Harmattan.

BOUCHE, Denise (1966). « Les écoles françaises au Soudan à l'époque de la conquête. 1884-1900 ». Cahiers d'études africaines, 22, 228-267.

BOUCHE, Denise (1975). L'Enseignement dans les territoires français de l'Afrique occidentale de 1817 à 1920. Mission civilisatrice ou formation d'une élite. Tome II. Service de reproduction des thèses, université de Lille.

CHAUBET, François (2006). La Politique culturelle française et la diplomatie de la langue.

L'Alliance française (1883-1940). Paris : L'Harmattan.

DELISLE, Philippe (dir.) (2009). L'Anticléricalisme dans les colonies françaises sous la Troisième République. Paris : Les Indes Savantes.

DUTEIL, Simon (2003). Parcours parallèles et construction d'une identité : les enseignants français dans les colonies françaises sous la Troisième République. Les enseignants français formés à l'École Jules Ferry de la Mission laïque française (1902-1914). Mémoire de D.E.A., université du Havre, multigr.

DUTEIL, Simon (2010). « Un instituteur colonial à Madagascar au début du XX $\mathrm{X}^{\mathrm{e}}$ siècle ». Histoire de l'éducation. En ligne : 128 | 2010, <http://histoireeducation.revues.org/2271> ; DOI : 10.4000/ histoire-education.2271, consulté le 01 janvier 2014.

KASPI, André (dir.) (2010). Histoire de l'Alliance israélite universelle, de 1860 à nos jours. Paris : Armand Colin.

LY, Boubacar (2009). Les Instituteurs au Sénégal. La condition d'enseignant et la vie sociale. Tome V. Paris : L'Harmattan.

MORLAT, Patrice (2009). « L'anticléricalisme en Indochine de 1900 à 1930 ». In Philippe Delisle, op. cit.

NISHIYAMA, Noriyuki (2005). « Cours de vacances de l'Alliance française sous la III République et genèse de la formation des maîtres étrangers de français ». Enseignement du français au Japon, 33.

NISHIYAMA, Noriyuki (2006). « La pédagogie bilingue de Louis Machuel et la politique du protectorat en Tunisie à la fin du XIX ${ }^{\mathrm{e}}$ siècle ». Revue japonaise de didactique du français, vol. 1 , $\mathrm{n}^{\circ} 1$.

SINGARAVÉLOU, Pierre (2011). Professer l'Empire. Les « sciences coloniales » en France sous la III République. Paris : Publications de la Sorbonne.

THEVENIN, André (2002). La Mission laïque à travers son histoire - 1902-2002. Paris : Mission laïque française. 
ZARATE, Geneviève (2000). « La reconnaissance des cultures indigènes ». Documents pour l'histoire du français langue étrangère ou seconde, 25. En ligne : 25 | 2000, <http:// dhfles.revues.org/2952>, consulté le 02 octobre 2015.

\section{NOTES}

1. Pour une présentation de la MLF, de son histoire, de son organisation, on pourra lire A. Thévenin 2002, auteur d'un ouvrage publié à l'occasion du centenaire de la création de l'association.

2. Sur l'histoire de l'Alliance israélite universelle, lire A. Kaspi 2010.

3. Voir F. Chaubet 2006.

4. L'étude la plus complète est constituée par le DEA de Simon Duteil (2003) consacré aux parcours et trajectoires des enseignants formés à l'EJF. L'École est aussi évoquée dans l'itinéraire de formation d'un instituteur colonial, Sosthène Penot, S. Duteil, 2010. L'École est encore brièvement citée par $\mathrm{D}$. Bouche dans sa thèse consacrée à l'enseignement dans les territoires d'Afrique occidentale (1974: 587), par P. Singaravélou (2011: 84-85) dans son ouvrage consacré aux spécialistes, aux établissements et aux multiples associations et sociétés savantes qui promeuvent une formation théorique ou pratique portant sur les colonies. A. Thévenin (op. cit.) y consacre aussi quelques pages dans son histoire de la MLF.

5. Voir D. Bouche 1966.

6. Ainsi du Congrès colonial international de Paris qui se tient du 30 juillet au 3 août 1889 qui voit s'opposer les partisans d'un enseignement assimilationniste et ceux d'un enseignement différencié.

7. Sur l'anticléricalisme dans les colonies, voir P. Delisle 2009.

8. Sur Pierre Deschamps, son idéal, sa carrière voir G. Zarate 2000.

9. Voir Noriyuki Nishiyama 2005.

10. L'EJF s'efforce de la sorte de trouver une réponse au problème de la dispersion géographique des territoires de l'empire en formant des instituteurs qui se destinent, au moins dans un premier temps, à exercer dans un territoire particulier. Les pratiques pédagogiques dans les colonies ne sont pas encore homogénéisées et sont laissées à l'initiative des directeurs locaux de l'enseignement.

11. Ainsi, Gustave Julien, professeur de malgache à l'École coloniale, intervient à différentes reprises pour présenter la civilisation malgache, en même temps que les boursiers de l'EJF sont invités à suivre ses cours de langue à l'École coloniale.

12. Ce programme, variable selon les années, est présenté dans la Revue de l'enseignement colonial à partir de 1904.

13. Le terme de « race » doit bien évidemment être entendu ici dans un sens autre que celui qui lui est donné aujourd'hui. Nous parlerions aujourd'hui de « civilisation ».

14. Sur cette question, voir plus particulièrement N. Nishiyama, 2006, qui situe plus exactement la pensée et les pratiques de Louis Machuel en la matière, méthodologie qui se rapproche quelque part, bien qu'il s'en défende, de celle de la leçon de choses, telle qu'elle est recommandée par les programmes de 1882 .

15. S. Duteil 2010.

16. À titre d'exemple, on peut relever les appuis suivants : Camille Guy, lieutenant-gouverneur du Sénégal fait attribuer en 1903 des bourses pour la création d'une section spéciale pour les futurs instituteurs du Sénégal, Gaston Doumergue, la même année, ministre des Colonies fait verser une subvention de 4000 francs pour financer 4 bourses, la Fondation Lucien Reinach verse 7000 francs en 1907. Mais les responsables de l'École ne peuvent s'empêcher de signaler la difficulté de la situation: "Nous aurons, cette année encore, de grands sacrifices à faire pour assurer 
l'existence de notre chère École; le service des bourses d'entretien nécessitera 12000 francs environ. Que nos amis se le disent.» (REC, 1904, 1, p. 22)

17. La MLF bénéficia en quelque sorte de ce que l'on appelle aujourd'hui une délégation de service public. Délégataire privé, la MLF pour autant n'était pas rattachée par un contrat clairement libellé aux instances publiques qui la finançaient.

18. Parcours professionnel restitué par L. Adler (1998) dans sa biographie de Marguerite Duras.

19. Dans son étude sur l'enseignement en Indochine, P. Bezançon (2002: 327) donne les chiffres suivants pour 1930 : 688 enseignants français pour 12014 enseignants indochinois. Même si la date est postérieure à la période qui nous intéresse ici, ces chiffres traduisent une tendance en place depuis de nombreuses années. L'affectation dans cette colonie d'enseignants formés par l'EJF ne pouvait apporter que des solutions ponctuelles. Selon d'autres études (P. Morlat, 2009 : 226), toujours en Indochine, pour ce qui est de l'instruction catholique au début des années vingt, on compte 350 pères qui s'y consacrent, une soixante de religieuses indigènes et un peu plus d'un millier de religieuses européennes, employées à l'éducation et aux œuvres de charité. Ce qui souligne la faiblesse, au moins numérique, de la contribution de l'EJF.

20. Ces instituteurs seront en effet placés dans un cadre spécifique, le cadre indigène, qui limite à la fois la progression dans les carrières et les rémunérations. Une revendication constante des instituteurs et moniteurs ainsi formés sera d'obtenir l'égalité de traitement avec les instituteurs venus de métropole. Voir pour le cas du Sénégal, Boubacar Ly 2009.

21. Même si par le traité de Versailles, elle se voit confier le mandat de gestion du Cameroun et du Togo, enlevés à l'Allemagne, ainsi que le mandat de la Syrie et du Liban au Proche-Orient.

\section{RÉSUMÉS}

L'extension considérable de l'espace colonial français à la fin du XIXe siècle, conduit les autorités françaises à créer en 1894 un ministère des Colonies, chargé de donner cohérence à l'action politique et administrative de la France dans ses colonies. La même année est fondée l'École coloniale dont la mission sera de former les administrateurs et les magistrats ayant à intervenir dans les colonies. Qu'en est-il des enseignants, notamment instituteurs, que l'on commence à envoyer dans ces mêmes territoires ? Un effort particulier sera-t-il entrepris en direction d'un corps de fonctionnaires ayant à intervenir auprès des publics d'enfants indigènes ? Il faut attendre en fait la création de l'École normale de l'enseignement colonial, qui sera appelée École normale Jules Ferry, par la Mission laïque française en 1902, pour voir se constituer un dispositif de formation spécifique, quasiment unique dans l'histoire de l'enseignement scolaire public dans les colonies. Cette école fonctionnera de 1902 à 1913 accueillant chaque année des promotions d'enseignants, en nombre limité, des instituteurs titulaires bénéficiant pour suivre cette formation de bourses, entre 10 et 15 instituteurs recrutés chaque année. Nous examinerons ici le programme de formation proposé à ces enseignants, dans une logique de préparation à l'exercice d'un métier nouveau, celui d'instituteur ayant à exercer dans les colonies, en comparant la formation ainsi dispensée à celle donnée dans les écoles normales d'instituteurs ordinaires et en la distinguant de celle qui pouvait concerner les missionnaires, appelés à la même époque à enseigner au-delà de leur mission première d'évangélisation. Nous nous appuierons à cet effet sur le corpus constitué par la Revue de l'enseignement colonial publiée par la Mission laïque française. 
The considerable extension of the French colonial area in the late nineteenth century, led the French authorities to create in 1894 a Colonial office, responsible for giving coherence to the political and administrative action of France in its colonies. In the same year was founded the Colonial School whose mission was to train administrators and magistrates for the colonies. What about teachers, including primary school teachers, who are now being sent to those territories? Will special efforts be devoted towards a body of officials having to work with indigenous children? With the creation of the École normale de l'enseignement colonial, later called École normale Jules Ferry, by the Mission laïque française in 1902, we see the birth of a specific training program, almost unique in the history of public school education for the colonies. This school will operate from 1902 to 1913, every year welcoming, with scholarships, 10 to 15 tenured teachers. We intend in this paper to describe the training program offered to these teachers, a program meant to prepare them for a new occupation: teacher in the colonies. In this paper we compare this program to the training offered in regular teacher training schools as well as to the training given to those missionaries who were called at the time to perform in the colonies more than mere evangelizing. We will rely on a corpus constituted by the Revue de l'enseignement colonial, a journal published by the Mission laïque française.

\section{INDEX}

Mots-clés : colonies, école normale, instituteurs, formation, missionnaires laïques.

Keywords : colonies, teacher training school, primary school teachers, training, secular missionaries.

\section{AUTEUR \\ GÉRARD VIGNER}

SIHFLES 